LBNL-61592

\title{
Performance Study of a Soft X-ray Harmonic Generation FEL Seeded with an EUV Laser Pulse
}

\author{
M. Gullans, J.S. Wurtele, G. Penn, A.A. Zholents
}

LBNL Technical Report: LBNL-61592

February 2007

\begin{abstract}
Disclaimer
This document was prepared as an account of work sponsored by the United States Government. While this document is believed to contain correct information, neither the United States Government nor any agency thereof, nor The Regents of the University of California, nor any of their employees, makes any warranty, express or implied, or assumes any legal responsibility for the accuracy, completeness, or usefulness of any information, apparatus, product, or process disclosed, or represents that its use would not infringe privately owned rights. Reference herein to any specific commercial product, process, or service by its trade name, trademark, manufacturer, or otherwise, does not necessarily constitute or imply its endorsement, recommendation, or favoring by the United States Government or any agency thereof, or The Regents of the University of California. The views and opinions of authors expressed herein do not necessarily state or reflect those of the United States Government or any agency thereof, or The Regents of the University of California.
\end{abstract}

Ernest Orlando Lawrence Berkeley National Laboratory is an equal opportunity employer.

This work was supported by the Director, Office of Science, High Energy Physics, U.S. Department of Energy under contract number DE-AC02-05CH11231 and grant DE-FG02-04ER41289. 


\title{
Performance study of a soft X-ray harmonic generation FEL seeded with an EUV laser pulse
}

\author{
M. Gullans ${ }^{\text {a }}$, J.S. Wurtele ${ }^{\mathrm{a}, \mathrm{b}}$, G. Penn ${ }^{\text {b,* }}$, A.A. Zholents ${ }^{\mathrm{b}}$ \\ ${ }^{a}$ University of California Berkeley, Berkeley, CA 94720, USA \\ ${ }^{\mathrm{b}}$ Lawrence Berkeley National Laboratory, Berkeley, CA 94720, USA
}

Received 29 November 2006; received in revised form 23 January 2007; accepted 2 February 2007

\begin{abstract}
The performance of a free electron laser (FEL) using a low-power extreme ultraviolet (EUV) pulse as an input seed is investigated. The parameters are appropriate for $30 \mathrm{~nm}$ seeds produced from high-power Ti:Sapphire pulses using high harmonic generation schemes. It is found that, for reasonable beam parameters, robust FEL performance can be obtained. Both time-independent and time-dependent simulations are performed for varying system parameters using the GENESIS simulation code. A comparison is made with a two-stage harmonic FEL that is seeded by a high-power Ti:Sapphire pulse.
\end{abstract}

(C) 2007 Elsevier B.V. All rights reserved.

PACS: 41.60.Cr; 29.27.-a

Keywords: HHG; Free electron laser

\section{Introduction}

The process of high-order harmonic generation (HHG) from a gas jet is now routinely used for the generation of bursts of coherent radiation at very short wavelengths [1, and references therein], with energies of the order of a few $n J$ per pulse. There is currently much anticipation at the use of these radiation sources as a seed for free electron lasers (FELs) which can produce extremely powerful pulses (with peak power on the order of a GW), with recent studies [2-5] considering output wavelengths ranging from $266 \mathrm{~nm}$ down to $12 \mathrm{~nm}$. Among the benefits of using an HHG source as an FEL seed, as opposed to operating in the mode of self-amplified spontaneous emission (SASE) [6], in which spontaneous radiation is amplified, are narrower (possibly transform-limited) output spectra, shotto-shot consistency, synchronization of the output pulse, and a shorter undulator section. Although the technology

\footnotetext{
* Corresponding author.

E-mail address: gepenn@lbl.gov (G. Penn).
}

of HHG sources is continually improving, currently the photon yield of experimental sources drops off sharply with photon energy at around a hundred $\mathrm{eV}$.

Seed pulses at longer wavelengths can be used to produce X-ray radiation using the technique of harmonic generation $[7,8]$, or even a harmonic cascade [9] consisting of multiple stages of harmonic generation. A harmonic generation FEL requires an initial seed pulse which is used to create an energy modulation on the beam. Many groups have investigated the use of conventional laser seed pulses in harmonic cascade FELs, including proposals for soft X-ray FELs based on this idea. By using an HHG seed in the extreme ultra-violet (EUV) wavelength range, the harmonic conversion to soft X-rays becomes much simpler and can be accomplished in a single stage. This option has been discussed previously [10,11], and a two-stage harmonic cascade yielding $0.3 \mathrm{~nm}$ radiation has also been analyzed [12].

In this paper, we consider an FEL which uses currently demonstrated HHG technology as a seed, with an available repetition rate of $1 \mathrm{kHz}$, to optimize the usefulness of the 
system as a research tool. We also seek to limit our attention to HHG sources which have been well-characterized and which have sufficient pulse energy to be effective as an FEL seed. A Ti:Sapphire laser driver containing a few $\mathrm{mJ}$ in a $\sim 25 \mathrm{fs}$ pulse at $800 \mathrm{~nm}$ wavelength has been shown to generate similarly short pulses at high harmonics down to $30 \mathrm{~nm}$ wavelength with a few $\mathrm{nJ}$ of energy per harmonic [1]. The repetition rate of these pulses is determined by the repetition rate of the Ti:Sapphire laser, and is of the order of $1 \mathrm{kHz}$. EUV pulses with pulse energy approaching $1 \mu \mathrm{J}$ have also been demonstrated, but at a much lower repetition rate [13].

We study two FEL designs, one using an EUV seed at $30 \mathrm{~nm}$ produced by $\mathrm{HHG}$, and the other a VUV seed at $240 \mathrm{~nm}$ using conventional nonlinear optics requiring a relatively modest drive laser that is also consistent with a $1 \mathrm{kHz}$ repetition rate. In both cases, the output from the FEL consists of soft X-rays in the "water window," having a wavelength of $3.75 \mathrm{~nm}$. This corresponds to the 8th harmonic of the EUV seed, using one stage of harmonic generation, or the 64th harmonic of the VUV seed, using two stages of harmonic generation. The electron beam has nominal parameters of $1 \mathrm{GeV}$ energy, $500 \mathrm{~A}$ current, $1.2 \mu \mathrm{m}$ normalized emittance, and an energy spread of $75 \mathrm{keV}$. We perform several optimizations for the HHG-seeded FEL based on variations of these parameters, and compare the requirements for the baseline FEL design with those for a conventionally-seeded FEL that produces similar output. In particular, we examine the sensitivity of the FEL output to noise in the input seed, and establish tolerances for the noise levels in order to achieve shot-to-shot repeatability and high longitudinal coherence. In simulations starting with a low-power seed, numerical noise tends to be greater than the expected shot noise, which is also modelled; we have sought out designs which result in the FEL simulation being relatively insensitive to numerically introduced noise.

The time-independent EUV-seeded FEL performance is presented in Section 2. Time-dependent simulation results are given Section 3. Results for an alternative FEL design that uses a $240 \mathrm{~nm}$ seed and two harmonic generation stages are given in Section 4. For the VUV-seeded FEL, the first stage of harmonic generation is used to produce a pulse of EUV radiation, also at $30 \mathrm{~nm}$, which is then used as input to the second stage of the FEL. The sensitivity to input noise for both FEL schemes is studied in Section 5, and a brief discussion of the results is given in the conclusions.

\section{Time-independent FEL simulations of an EUV-seeded FEL}

A schematic of the FEL is depicted in Fig. 1. We assume that the EUV seed pulse has a central wavelength of $30 \mathrm{~nm}$, a pulse duration of $25 \mathrm{fs}$ (FWHM in power), a peak power of $100 \mathrm{~kW}$ and has transverse and temporal coherence. We consider an HHG pulse that has already been filtered both in terms of selecting a single harmonic and of smoothing

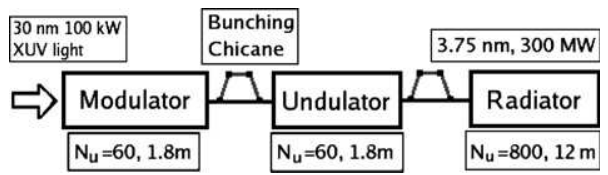

Fig. 1. Schematic of an FEL seeded by a $30 \mathrm{~nm}$ EUV pulse. The first two undulators are tuned for $30 \mathrm{~nm}$, and have 60 periods and a $3 \mathrm{~cm}$ wavelength. The final radiator is tuned for the eighth harmonic, has an undulator period of $1.5 \mathrm{~cm}$, and is $12 \mathrm{~m}$ long ( 800 periods). The breaks between undulators are each $1 \mathrm{~m}$ long and contain magnetic chicanes.

the sub-femtosecond timing structure. We also note that interaction of the first undulator in the FEL with the HHG pulse should not be sensitive to variations on such short time scales. The electron beam enters from the left into an optical klystron [14] consisting of two undulators separated by a bunching chicane. Following a second bunching chicane, the electron beam radiates in the final undulator at a harmonic of the HHG seed. The laser seed is focused to a waist of $120 \mu \mathrm{m}$ radius in the midpoint of the first undulator, corresponding to a Rayleigh length of $3 \mathrm{~m}$. The nominal electron beam is as described above. The typical electron beam radius is $60 \mu \mathrm{m}$.

The FEL consists of three undulators, separated by $1 \mathrm{~m}$ breaks which contain a magnetic chicane to generate or enhance electron bunching. The first two undulators are tuned for the $30 \mathrm{~nm}$ wavelength of the HHG pulse, and each have a $3 \mathrm{~cm}$ wavelength with 60 periods, for a length of $1.8 \mathrm{~m}$. The third undulator is tuned to the 8 th harmonic, or $3.75 \mathrm{~nm}$ wavelength, and has a $1.5 \mathrm{~cm}$ wavelength with a total of 800 periods, for a total length of $12 \mathrm{~m}$. The bunching chicane following the modulator has an $R_{56}$ of $30 \mu \mathrm{m}$, while the chicane before the final radiator has $R_{56}=$ $7.5 \mu \mathrm{m}$.

The EUV seed and the electrons overlap in the first undulator, creating an energy modulation. The chicane is used to convert the energy modulation into a current modulation, which generates further energy modulation in the second undulator. This optical klystron configuration results in much stronger modulation of the electron beam compared to the case where the same total length of undulator is used without a magnetic chicane. Furthermore, in this way the modulator is kept sufficiently short that diffraction of the HHG signal does not negatively impact the performance. By the end of the second undulator, the HHG signal at the original wavelength is amplified to $27 \mathrm{MW}$, and the electron bunching at the fundamental is 0.14 . The modulated beam leaves the second undulator and is further bunched by another magnetic chicane in order to maximize the bunching at the eighth harmonic, achieving a bunching of 0.04 . It then enters the radiator which is tuned to a wavelength of $3.75 \mathrm{~nm}$. Except for the use of the optical klystron, this design is similar to earlier designs for harmonic generation in FELs. An output power of approximately $300 \mathrm{MW}$ is produced.

Numerical simulations were performed using GENESIS [15], which allows for either single-slice (i.e., time-indepen- 
dent) or time-dependent simulations. The resonance condition [16] is $\lambda \approx \lambda_{\mathrm{u}}\left(1+a_{\mathrm{u}}^{2}\right) / 2 \gamma^{2}$, where $\lambda$ is the radiation wavelength, $\gamma$ is $E / m_{\mathrm{e}} c^{2}, E$ is the electron beam energy, $m_{\mathrm{e}}$ the electron mass, $c$ is the speed of light, $a_{\mathrm{u}} \equiv$ $e B_{\mathrm{rms}} \lambda_{\mathrm{u}} / 2 \pi m_{\mathrm{e}} c$ is the normalized undulator parameter, $\lambda_{\mathrm{u}}$ is the undulator period, $B_{\mathrm{rms}}$ is the rms magnetic field in the undulator, and $|e|$ is the electron charge. FEL tuning is accomplished by adjusting $a_{\mathrm{u}}$ so as to maximize FEL output power.

The initial FEL designs were optimized with single-slice simulations, and then the single slice optimum configurations were used in full time-dependent runs. The first two undulators are shorter than or comparable to the gain length, which is predicted to be $0.8 \mathrm{~m}$ compared to $1.1 \mathrm{~m}$ for the third undulator. Therefore, they operate in the low-gain regime, and radiation due to amplification of spontaneous emission is small. The seeded bunching is well above noise levels in these simulations. Care was taken to avoid numerical problems that arise from the low power of the initial laser seed. For example, at energy spreads larger than the nominal value, the weak initial energy modulation and the large $R_{56}$ required to achieve bunching resulted in large statistical fluctuations in the distribution of macroparticles and output power. We note that the "quiet load" algorithm, which is designed to reduce spurious frequency components in the electron distribution, may fail due to the fact that the required amplification of the initial laser seed generates statistical noise in the macroparticles. Design of seeded FELs always requires attention to be paid to competition between the seeded interaction and spontaneous emission from fluctuations within the electron beam. In the examples considered here, special care must be taken to ensure that the simulation results have converged numerically. Because of computational limitations, this imposes constraints not only on the complexity of the simulation but also on electron beam quality and input seed parameters. The nominal seed power of $100 \mathrm{~kW}(2.5 \mathrm{~nJ})$ is already low by FEL standards, and we have assumed optimal focusing of the HHG seed. For the nominal set of parameters, convergence tests show computational fluctuations of $4 \%$ for the output power, while the output phase fluctuates by $0.15 \mathrm{rad}$.

We evaluated the performance with transverse emittance $\epsilon_{N}$ of $1.2 \mu \mathrm{m}, 1.0 \mu \mathrm{m}$, and $0.8 \mu \mathrm{m}$, adjusting the beam radius to match a beta function of around $6 \mathrm{~m}$. We also considered increasing the peak current, possibly at the expense of increasing the energy spread $(I=600 \mathrm{~A}$ with either $\sigma_{\mathrm{E}}=75 \mathrm{keV}$ or $\left.\sigma_{\mathrm{E}}=100 \mathrm{keV}\right)$. The results are shown in Fig. 2, where the logarithmic power is plotted as a function of interaction length in the final undulator. These results are summarized in Table 1 . The cases were individually optimized by varying $a_{\mathrm{u}}$ in each undulator as well as the strength of the $R_{56}$ parameter in the magnetic chicane. Three of the curves in Fig. 2 illustrate the emittance sensitivity; also shown is the performance which could be achieved for a peak current of $600 \mathrm{~A}$, both with

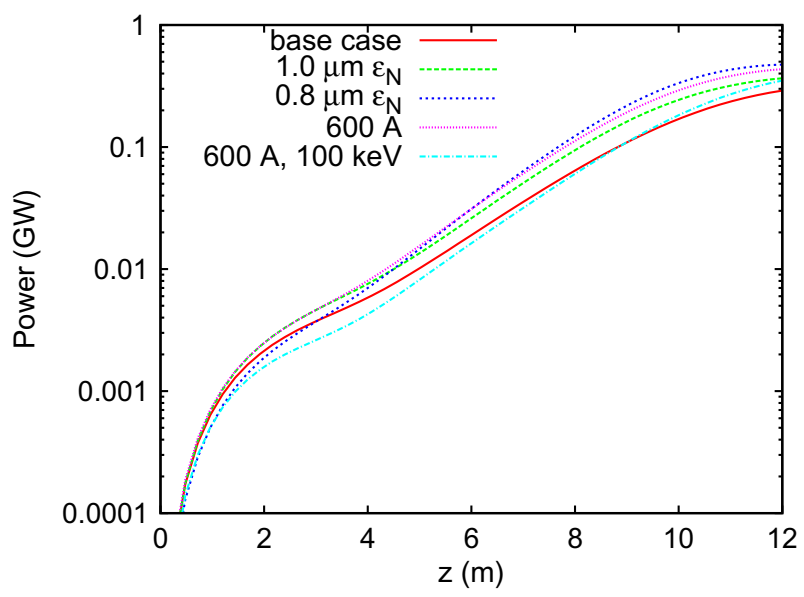

Fig. 2. Power as a function of interaction length along the final radiator for different beam parameters.

Table 1

Sensitivity of FEL performance, after tuning, to parameter variations in time-independent simulations

\begin{tabular}{llllc}
\hline Beam parameters & $\begin{array}{l}\text { Initial } \\
\text { bunch }(\%)\end{array}$ & $\begin{array}{l}\text { Power: } 2 \mathrm{~m} \\
(\mathrm{MW})\end{array}$ & $\begin{array}{l}\text { Power: end } \\
(\mathrm{MW})\end{array}$ & $\begin{array}{l}L_{\mathrm{G}} \\
(\mathrm{m})\end{array}$ \\
\hline Nominal & 4.3 & 2.0 & 290 & 1.7 \\
$\epsilon_{N}=1.0 \mu \mathrm{m}$ & 4.1 & 2.3 & 366 & 1.6 \\
$\epsilon_{N}=0.8 \mu \mathrm{m}$ & 3.3 & 1.8 & 474 & 1.4 \\
$I=600 \mathrm{~A}$ & 3.7 & 2.3 & 435 & 1.5 \\
$I=600 \mathrm{~A}$ and & 3.0 & 1.5 & 350 & 1.5 \\
$\quad \sigma_{\mathrm{E}}=100 \mathrm{keV}$ & & & &
\end{tabular}

The nominal parameters are $\epsilon_{N}=1.2 \mu \mathrm{m}, E=1.0 \mathrm{GeV}, I=500 \mathrm{~A}$, and $\sigma_{\mathrm{E}}=75 \mathrm{keV}$

and without a corresponding increase in energy spread to $100 \mathrm{keV}$. For the nominal parameters, the initial bunching is $4.3 \%$, the power at $2 \mathrm{~m}$ is $2.0 \mathrm{MW}$, the peak power is $290 \mathrm{MW}$ and the gain length $L_{\mathrm{G}}$ is $1.7 \mathrm{~m}$, where $1 / L_{\mathrm{G}}$ is the typical value of $(1 / P) \mathrm{d} P / \mathrm{d} z$ during the period of exponential growth in output power. This gain length corre-

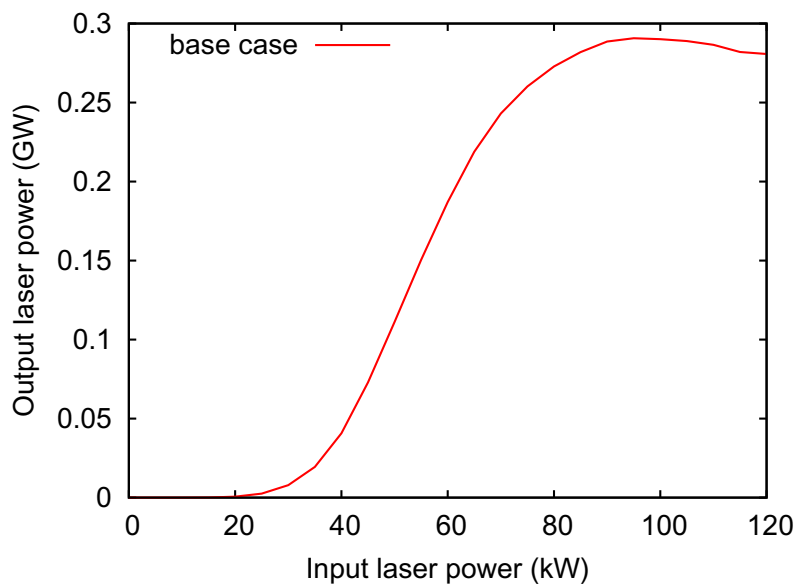

Fig. 3. The output power as a function of the input power, tuned for a $\sim 100 \mathrm{~kW}$ input, for the nominal FEL parameters. 
sponds to a Pierce parameter [17] of $4 \times 10^{-4}$, and is worse than the idealized gain length of $1.1 \mathrm{~m}$ as a result of the increase in energy spread to $315 \mathrm{keV}$ while passing through the first two undulators.

We see from Fig. 2 that the FEL is operating in the highgain regime. In each simulation, the power initially has quadratic growth determined by the initial bunching and then begins to grow exponentially, with gain length $L_{\mathrm{G}}$, at around $3 \mathrm{~m}$ in the undulator. The bunching reaches a maximum and then begins to decrease, leading to the saturation of power growth by the end of the radiator. A slight over-modulation of the electron beam, in few instances, causes some debunching and reduction of power growth just as the exponential growth becomes dominant. These results show some interesting tradeoffs in achieved performance among different electron beam parameters. Reducing $\epsilon_{N}$ reduces the gain length and, as the final undulator is much longer than the gain length, this yields a strong improvement in final output power, The nominal $1.2 \mu \mathrm{m}$ emittance can be used to achieve the same performance as that of the $1.0 \mu \mathrm{m}$ emittance beam by taking the additional step of compressing the beam to increase the current to $600 \mathrm{~A}$, allowing for a proportional increase in energy spread.

The dependence of the performance on the input laser power is seen in Fig. 3. As usual for a harmonic generation scheme, the output power drops essentially to zero at some cutoff power (here, around $20 \mathrm{~kW}$ ). The output power is fairly insensitive to the input laser power down to $75 \mathrm{~kW}$, and a $10 \%$ variation in laser power leads to a decrease in the peak output power (centered at $95 \mathrm{~kW}$ ) of only $5 \%$. These results give a rough idea of the temporal shape of the output pulse given the input laser seed, which will be studied more accurately below using full time-dependent simulations.

\section{Time-dependent simulations}

Time-dependent FEL simulations were performed for a uniform beam profile and a Gaussian HHG laser seed with a $25 \mathrm{fs}$ FWHM and peak power of $100 \mathrm{~kW}$. The actual output from HHG sources consists of a series of short spikes separated by half of the fundamental laser period; the typical wavelength of the fundamental laser is $800 \mathrm{~nm}$ [1]. However, the specialized optics required for the transport of $30 \mathrm{~nm}$ radiation from the HHG source to the FEL may smooth out this temporal structure, and the FEL interaction itself washes out rapid time structures by a combination of frequency selection and slippage between the radiation field and the electron beam, as shown in Refs. $[2,18]$. In these simulations we neglect the microstructure within the HHG pulse and treat it as a smooth Gaussian with frequency content restricted to the desired harmonic. Neither the temporal envelope nor the transverse profile of measured HHG pulses are necessarily Gaussian in practice [2,19], but are taken as such to isolate the effect of noise in the input seed.
The peak laser power overlaps the electron beam at $t=0$ on the horizontal axis in Fig. 4. The displacement of the final output is due to slippage of the electron beam with respect to the laser fields. The studies were carried out using all the nominal parameters except for $\epsilon_{N}$, which had values of $1.2,1.0$, and $0.8 \mu \mathrm{m}$. These cases were previously optimized for output power based on single-slice simulations. Table 2 and Fig. 4 summarize the results.

There are several qualitative similarities in the power plots as shown Fig. 4. Foremost is the asymmetry in the FEL output, with peak power at the leading edge of the pulse that is even more than predicted from single-slice simulations. The average power within the core of the pulse is in reasonable agreement with the single-slice simulation results of Table 1 . This feature occurs even in the absence of HHG fluctuations or shot noise in the electron beam. The ubiquitous appearance of this feature suggests that this is an intrinsic characteristic of this FEL scheme when seeded with such a short Gaussian laser pulse. When the slippage length and pulse width are comparable to each other, phase variations across the pulse resulting from the
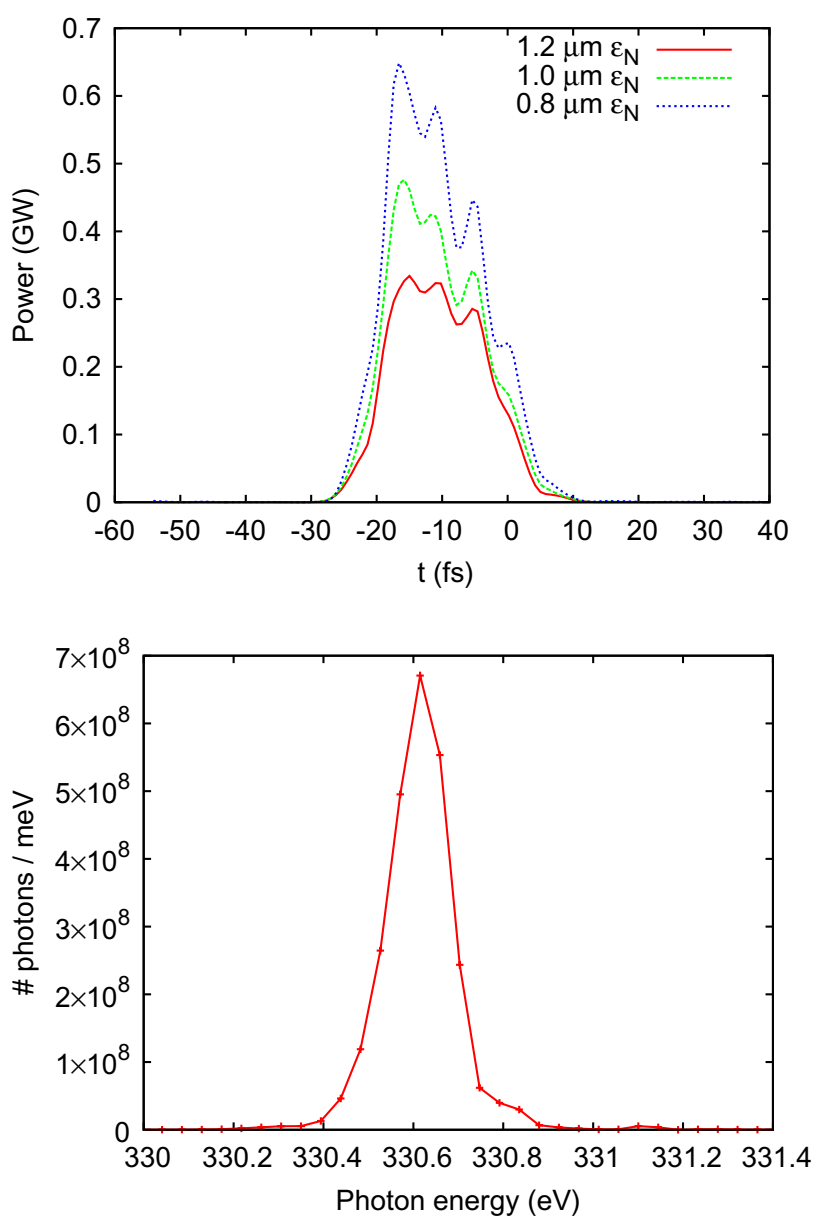

Fig. 4. The top graph shows the power profile at the end of the FEL as a function of longitudinal position for various examples, while the bottom graph shows the spectrum for the $1.2 \mu \mathrm{m} \epsilon_{N}$ case. The spectra in the other examples are similar except for their magnitude, as is expected since the spread in frequency is largely determined by short duration of the pulse. 
Table 2

Summary of the time-dependent simulation results

\begin{tabular}{lll}
\hline Setup $(\mu \mathrm{m})$ & Peak power $(\mathrm{MW})$ & FWHM in spectrum $(\%)$ \\
\hline$\epsilon_{N}=1.2$ & 310 & 0.046 \\
$\epsilon_{N}=1.0$ & 460 & 0.045 \\
$\epsilon_{N}=0.8$ & 660 & 0.043 \\
\hline
\end{tabular}

variation in initial seed power act as an effective detuning [20], which in some portions of the pulse enhances FEL amplification and in other portions interferes. The optical klystron configuration appears to enhance this effect. Also notable is the dramatic rise in peak power between the 1.2 and $0.8 \mu \mathrm{m}$ emittance cases, by slightly more than a factor of two. This confirms that the single-slice runs give a good indication of FEL behavior.

To estimate the longitudinal coherence of the output laser pulse we can compare the expected FWHM in the spectrum, assuming a perfect Gaussian output power profile with the same duration (in terms of FWHM) as measured in the top graph of Fig. 4, to the observed FWHM in the spectrum. An ideal Gaussian pulse is used for comparison to include both the effects of phase variations and fluctuations in output power, both of which are indicative of a loss of longitudinal coherence. The expected FWHM in the spectrum for a Gaussian output profile is given by $\Delta \lambda / \lambda_{0}=0.44 \lambda_{0} / c \Delta t$. Here, $\lambda_{0}$ is the nominal wavelength ( $3.75 \mathrm{~nm}$ in our case), $\Delta t$ is the FWHM of the power profile, and $\Delta \lambda$ is the expected FWHM of the spectrum. In Fig. 4, we see that the FWHM of the power profile is about 20 fs for each case, which gives $\Delta \lambda / \lambda_{0}=0.028 \%$. In the observed spectrum we have a FWHM of about $0.05 \%$, which is a factor of 1.8 times larger than the calculated result. This is a reasonable result for the longitudinal coherence, since our output power profile has two or more peaks in each case. We can do a similar calculation for an output power profile given by a square pulse $20 \mathrm{fs}$ long with no phase variation, which yields an expected FWHM of $0.056 \%$. There is in fact very little phase variation, approximately $1 \mathrm{rad}$, across the output pulse.

\section{Comparison to a two-stage FEL seeded at $240 \mathrm{~nm}$}

We now consider an alternative FEL design using a $240 \mathrm{~nm}$ wavelength laser seed with a peak power of 100 MW. This FEL, as seen in Fig. 5, incorporates two harmonic stages to reach the same final wavelength. Each stage consists of a modulator, a magnetic chicane, and a radiator, and a "fresh-bunch" approach [21] is used between stages, so that the radiation produced at the first stage (at the 8th harmonic of $240 \mathrm{~nm}$, or $30 \mathrm{~nm}$ ) subsequently overlaps a previously unmodulated section of the electron bunch. The first undulator has a period of $12 \mathrm{~cm}$, and is $2.4 \mathrm{~m}$ long. Its gain length is $1.6 \mathrm{~m}$. After a magnetic chicane with $R_{56}=18 \mu \mathrm{m}$, the electrons enter the first radiator. Starting with a laser pulse of $100 \mathrm{MW}$, the initial bunching in the final radiator is $6.4 \%$, and the

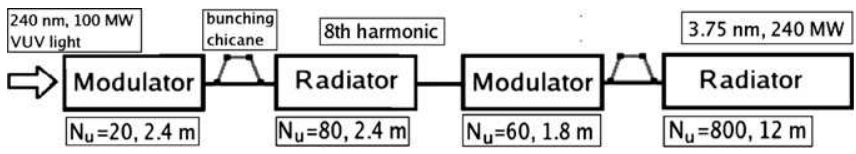

Fig. 5. Schematic of an FEL seeded by a $240 \mathrm{~nm}$ VUV pulse. The first undulator is tuned for $240 \mathrm{~nm}$, has 20 periods and a $12 \mathrm{~cm}$ wavelength. The next two undulators are tuned for $30 \mathrm{~nm}$, have 80 and 60 periods respectively, and a $3 \mathrm{~cm}$ wavelength. The final radiator has a $1.5 \mathrm{~cm}$ wavelength and is $12 \mathrm{~m}$ long ( 800 periods). The breaks between undulators are each $1 \mathrm{~m}$ long and contain either a magnetic chicane for bunching, or a "fresh-bunch" delay section for the center break.

final output power is $236 \mathrm{MW}$. Time-dependent results are shown below in Fig. 6. In order to yield an output pulse with similar characteristics to that of the EUV-seeded FEL, the pulse duration of the laser seed is taken to be $45 \mathrm{fs}$ FWHM, for a total pulse energy of $4.8 \mu \mathrm{J}$. The increased peaking of the output pulse is due to the additional nonlinearity introduced by the first stage, where the signal is upshifted from a $240 \mathrm{~nm}$ wavelength to a $30 \mathrm{~nm}$ wavelength. Furthermore, the slippage in the first undulator is much greater than in the HHG-seeded examples (16 fs compared to $6 \mathrm{fs}$ ), so that a $25 \mathrm{fs}$ pulse would exhibit even stronger time-dependent effects.
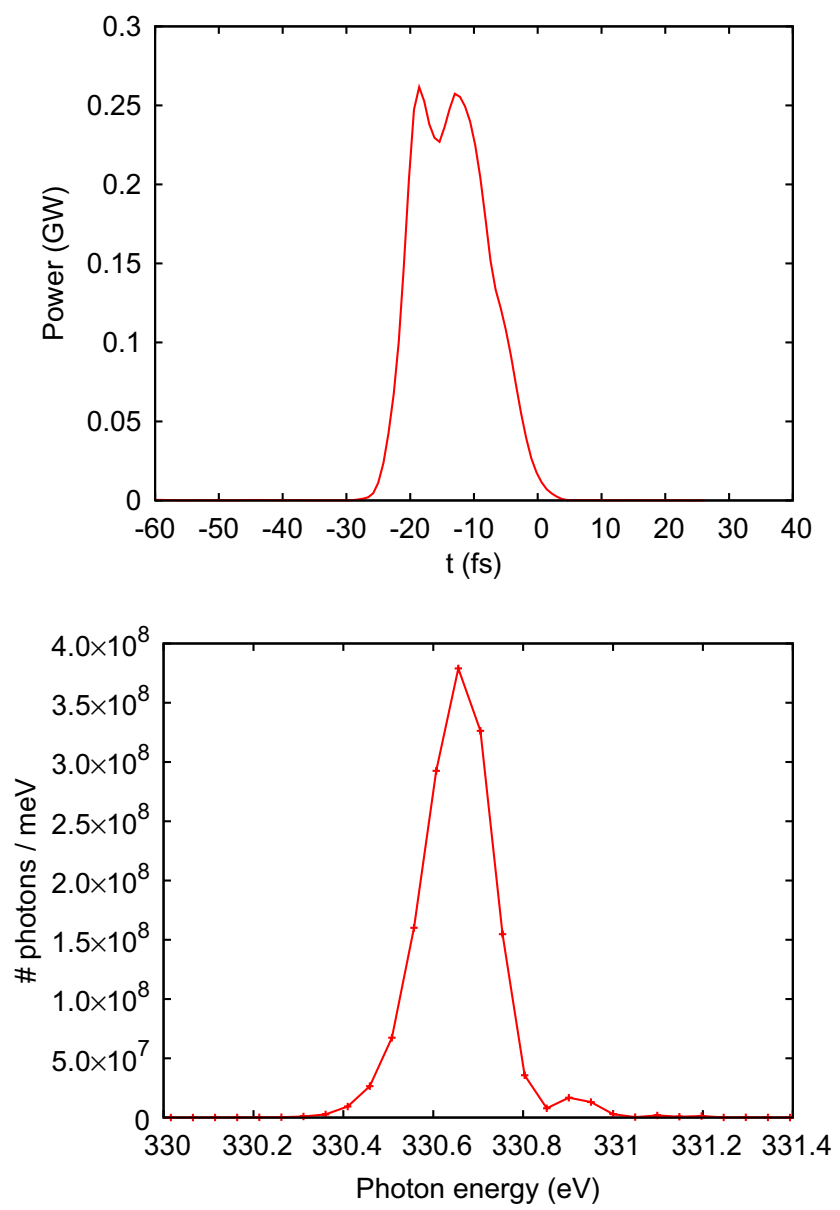

Fig. 6. Power as a function of time (top) and spectrum (bottom) for a $3.75 \mathrm{~nm}$ FEL seeded by a $240 \mathrm{~nm}$ laser pulse. 
The output from this FEL is, by design, closely similar to that of the HHG-seeded FEL, although the peak output power is slightly lower. The example using an HHG seed has a more complex power profile, while the main feature for this case is a slight dip in power near the center of the output pulse. This is probably a consequence of a curve similar to that of Fig. 3, where the peak of the laser seed induces a larger than optimal energy modulation. Both configurations end with a similar $12 \mathrm{~m}$ long final undulator. The FEL seeded with an HHG source requires the HHG system including a very high-power laser, and an additional $3.6 \mathrm{~m}$ of undulator; the FEL seeded with a VUV laser requires a more modest laser and a conventional means for shifting to a shorter wavelength, for example an optical parametric amplifier, and an additional $6.6 \mathrm{~m}$ of undulator. The use of fresh-bunch technique for the VUV-seeded FEL also imposes additional constraints on synchronization, although for the short pulses considered this should not be an issue.

Laser seeds having different input power can be accomodated by varying the length of the initial modulator. The length of modulator should approximately scale as the inverse square root of the peak power to yield the same output power. This scaling holds reasonably well even when the spot size of the laser seed is kept fixed, until the modulator becomes sufficiently long that either the laser seed becomes amplified, or diffraction reduces the overlap between the laser and electron beams. For the case of an FEL seeded by a conventional, $240 \mathrm{~nm}$ wavelength laser, the required length of modulator follows the above scaling for peak powers which vary by an order of magnitude from the nominal value of $100 \mathrm{MW}$. For the nominal EUV seed power of $100 \mathrm{~kW}$, the modulator is $1.8 \mathrm{~m}$ long; the above scaling applies for higher seed power, but with $25 \mathrm{~kW}$ peak power, the modulator only needs to be $3.0 \mathrm{~m}$ long due to amplification of the seed within the modulator. It appears that the limiting factor on the ability to use a low-power EUV signal is competition from exponential growth of noise rather than any inability to sufficiently modulate the beam, although for short-duration pulses there will be stronger effects of slippage as longer modulators are used. The gain length for the first modulator is $0.8 \mathrm{~m}$, and in the $25 \mathrm{~kW}$ example the laser seed is amplified to $100 \mathrm{~kW}$ within $\approx 3 \mathrm{~m}$ of undulator; shot noise in the electron beam as well as any noise in the seed laser will be similarly amplified. Competition between amplification of the input seed with amplification of shot noise through SASE sets a minimum power level for the input seed. This is especially true for short seed pulses, where only a small fraction of the electron beam may be exposed to the input seed. All time-dependent simulations presented in this paper include the standard model provided by GENESIS for shot noise within the electron beam distribution, although the parameters for these FEL configurations have been designed to be fairly insensitive to the expected levels of shot noise.

\section{Sensitivity to input laser noise}

We now consider the sensitivity of the FEL to noise in the input laser seed. As little is known about the power and phase fluctuations of HHG sources, and as different conventional laser systems exhibit various noise characteristics, a generic noise model is used to estimate tolerance levels for the two FEL designs. Noise can originate either in the initial drive laser or in the optical manipulations which need to be performed downstream. The noise model used is a flat power spectrum within some interval around the laser seed frequency. Simulations incorporating this noise model are used to determine the level of noise at which significant fluctuations in the output spectrum or power profile result. This serves as a rough indicator of the seed quality required to achieve high shot-to-shot repeatability. Note that more coherent forms of jitter, such as variations in peak power or energy per pulse, are not considered except insofar as they may result from incoherent noise.

Due to the resolution and time-window of the simulations, the noise levels are modelled as a fixed number of frequency bins, each of which have constant power and random phase. The electric field profile resulting from this spectrum is then added to the electric field of the ideal laser. Because the frequency components far from the resonant wavelength are not expected to have much of an effect on the FEL, we describe the noise levels in terms of power per $0.1 \%$ bandwidth relative to the resonant wavelength. For reference, the HHG seed at $30 \mathrm{~nm}$ with $100 \mathrm{~kW}$ peak power and 25 fs FWHM has a peak longitudinal photon density in optical phase space of $53 \mathrm{~kW}$ per $0.1 \%$ bandwidth. The $240 \mathrm{~nm}$ laser with $100 \mathrm{MW}$ peak power and $45 \mathrm{fs}$ FWHM has a peak longitudinal photon density of $12.0 \mathrm{MW}$ per $0.1 \%$ bandwidth. In the figures below, the example power profiles for the seed including noise are smoothed over a $5 \mathrm{fs}$ time scale, which is slightly longer than the slippage during the first undulator. Examining the noise on a finer grid only adds spikes which are not in fact resolved by the electrons themselves. Separating the radiation into signal and noise terms, the harmonic generation process is expected to degrade the signal to noise ratio by a factor of $N^{2}$ compared to the input signal, where $N$ is the total harmonic conversion factor $[22,23]$. Because increased phase incoherence is an important aspect of the harmonic conversion process, the effect of the noise is more apparent in the spectrum than in the power profile. This enhancement in noise from the harmonic amplification process implies that even a nearly transform-limited input pulse can yield longitudinally incoherent output at a high harmonic. This effect not only applies to harmonic generation in an FEL, but to the HHG process and to the process (for example, optical parametric amplification) used to produce the VUV laser from a drive laser that is also typically a Ti:Sapphire laser at $800 \mathrm{~nm}$. Thus, HHG pulses would be expected to have a lower signal to noise ratio than VUV pulses produced from similar drive lasers 


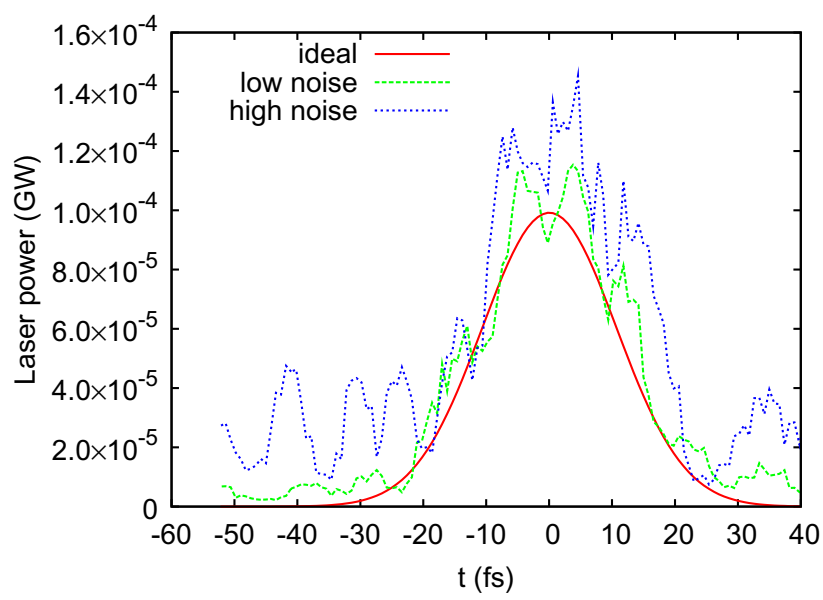

Fig. 7. Examples of HHG seed power profiles, ideal (no noise) and with noise levels of $50 \mathrm{~W}$ and $200 \mathrm{~W}$ per $0.1 \%$ bandwidth.

simply due to the higher harmonic amplification factor. However, as the total harmonic factor from the drive laser to water window wavelengths is the same, noise in the drive laser should in principle have a similar effect in either configuration, unless other sources of optical noise are present.
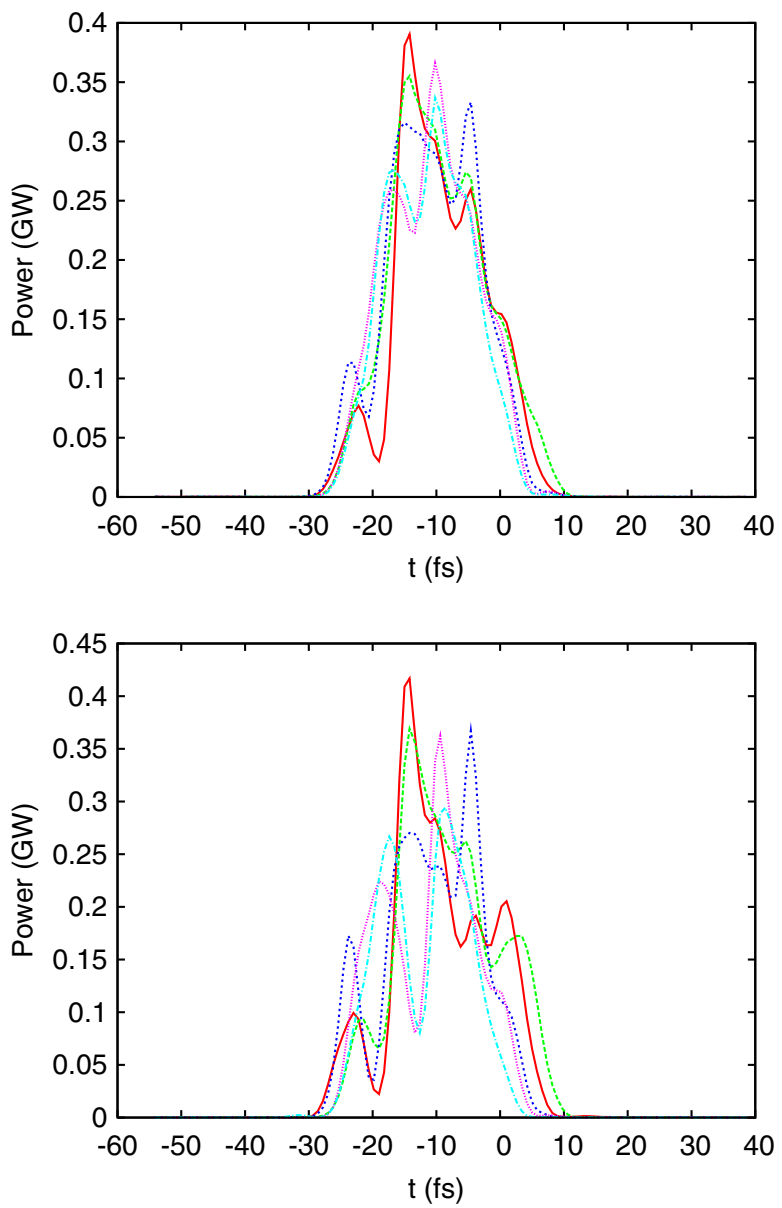

For the nominal example using an EUV seed, significant distortions in the output profile and spectrum are apparent when the noise level is of the order of $200 \mathrm{~W}$ per $0.1 \%$ bandwidth. This is a factor of approximately 250 below the signal in terms of longitudinal photon density in optical phase space, although the noise is taken to have a much broader spectrum. Fig. 7 shows examples of the laser seed in the presence of noise levels of $50 \mathrm{~W}$ and $200 \mathrm{~W}$ per $0.1 \%$ bandwidth. The resulting output at $3.75 \mathrm{~nm}$ is shown in Fig. 8 for multiple test cases; for a given noise level, each test case corresponds to a different random number seed used to generate the input noise. For this FEL design, the fluctuations in the power profile due to noise are comparable to the fluctuations in the spectrum.

For an FEL seeded with a $240 \mathrm{~nm}$ laser, significant distortions in the output are apparent when the noise level is of the order of $1 \mathrm{~kW}$ per $0.1 \%$ bandwidth. This is a factor of approximately 12,000 below the signal. The much larger factor than in the HHG-seeded FEL is largely explained by the factor of 64 in wavelength between the laser seed and the output, compared to a factor of 8 in the previous case. Through the harmonic conversion process, the signal to noise ratio is expected to degrade by the square of the total harmonic multiplication factor. This enhancement in noise
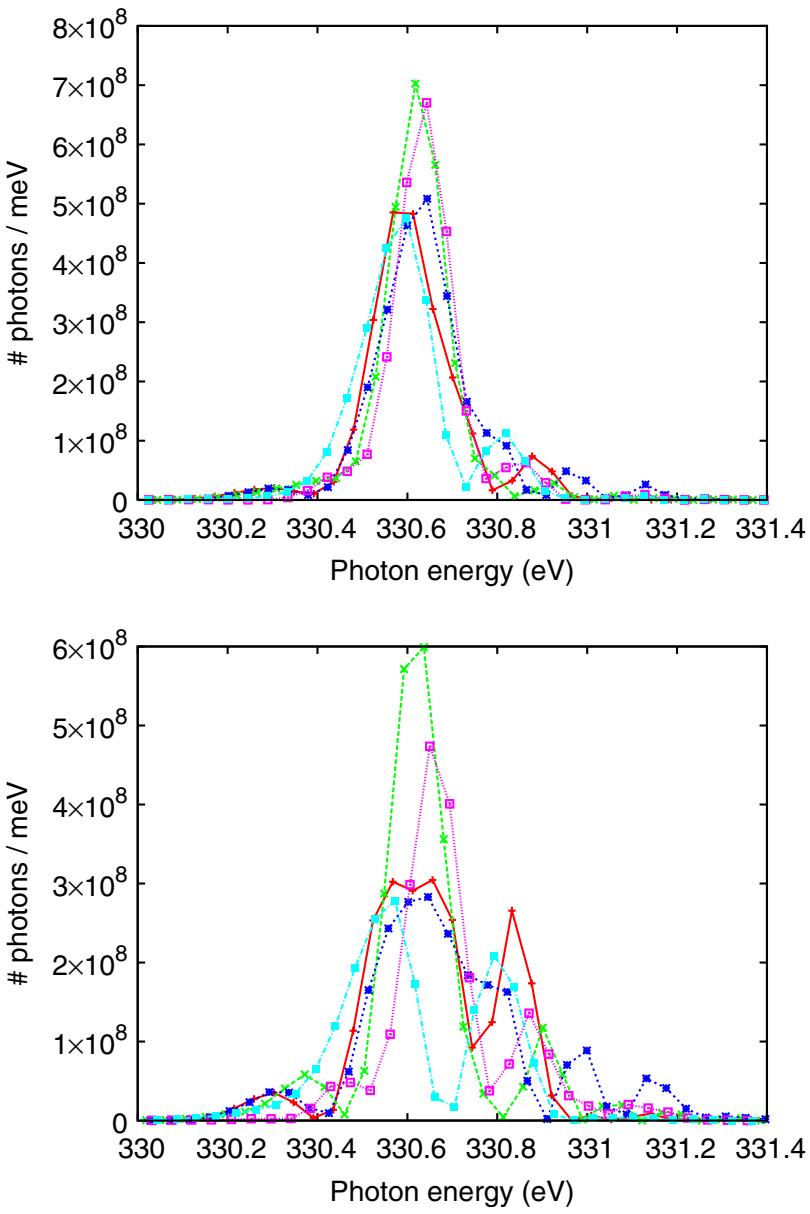

Fig. 8. Power profile and spectrum of multiple examples of an HHG-seeded FEL with noise levels of $50 \mathrm{~W}$ (top) and $200 \mathrm{~W}$ (bottom) per $0.1 \%$ bandwidth. Each curve corresponds to a different random number seed used to generate the input laser noise. 


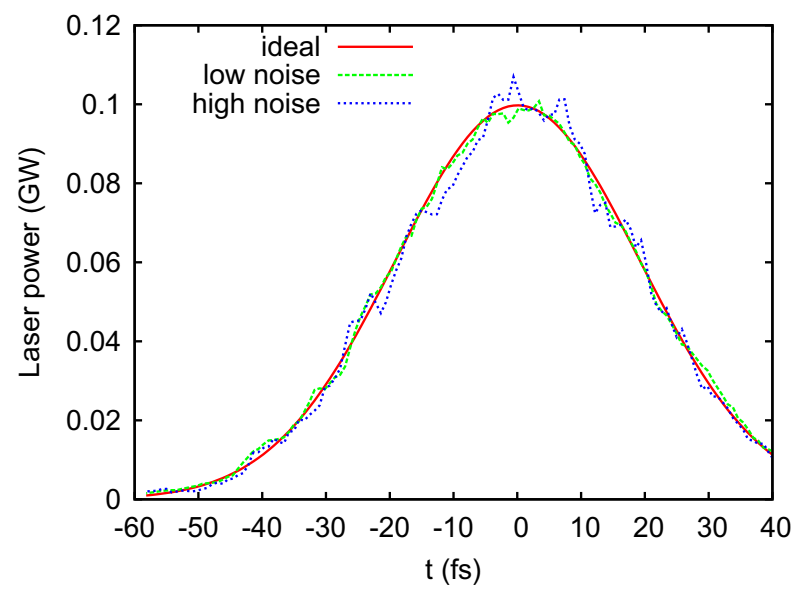

Fig. 9. Examples of $240 \mathrm{~nm}$ laser seed power profiles, ideal (no noise) and with noise levels of $250 \mathrm{~W}$ and $1 \mathrm{~kW}$ per $0.1 \%$ bandwidth.

will be characterized as a larger effective longitudinal photon density for the initial seed noise. Thus, for the "highnoise" HHG-seeded example, the effective initial noise level is estimated to be $12.8 \mathrm{~kW}$ per $0.1 \%$ bandwidth, or $24 \%$ of the signal. For the "high-noise" conventional laser example, the effective input noise level is estimated to be
4.1 MW per $0.1 \%$ bandwidth, or $34 \%$ of the signal. Note that for the FEL seeded with a $240 \mathrm{~nm}$ laser, the fluctuations in the power profile due to noise are much weaker than the fluctuations in the spectrum. The character of the noise enhancement produced by harmonic conversion emphasizes phase noise over power fluctuations at high harmonics.

Fig. 9 shows examples of the laser seed in the presence of noise levels of $250 \mathrm{~W}$ and $1 \mathrm{~kW}$ per $0.1 \%$ bandwidth. The resulting output at $3.75 \mathrm{~nm}$ is shown in Fig. 10. The seed duration is longer, with a FWHM of $45 \mathrm{fs}$, to accomodate the extra narrowing incurred by the additional harmonic stage. It is notable that noise levels corresponding to a significant degradation in the output are characterized by a much weaker distortion in the input seed laser than the analogous examples for the HHG seed. The difference between the power profile and spectrum in terms of sensitivity to noise is also more prominent than in the EUV-seeded FEL. For the example with $250 \mathrm{~W}$ per $0.1 \%$ bandwidth of noise, the power profile is very well-defined except for fluctuations in the peak power, whereas the spectrum already has significant fluctuations not only in width but in the central wavelength as well. The large overall jump to the 64th harmonic
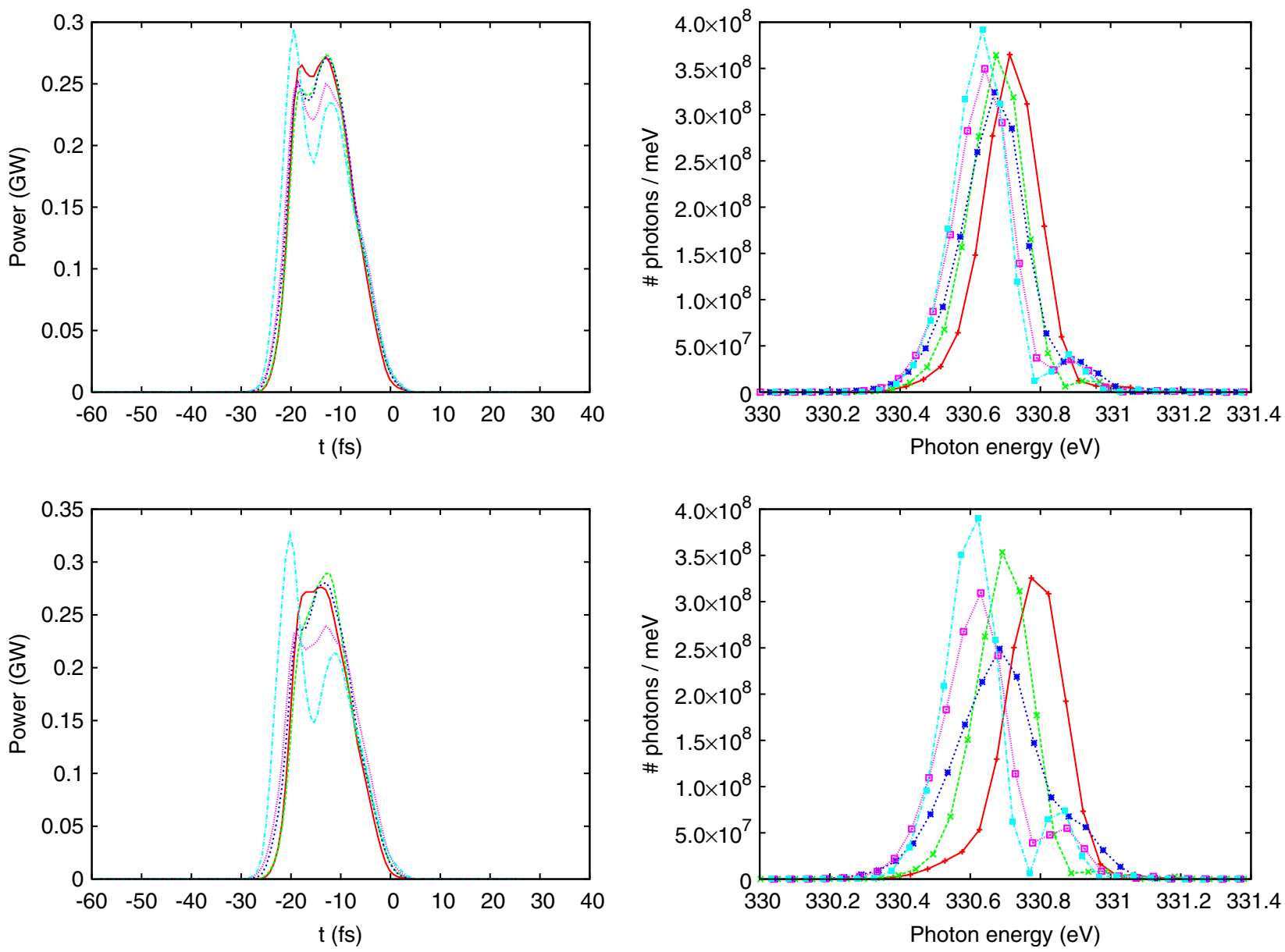

Fig. 10. Power profile and spectrum of multiple examples of an FEL seeded with a $240 \mathrm{~nm}$ laser with noise levels of $250 \mathrm{~W}$ (top) and $1 \mathrm{~kW}$ (bottom) per $0.1 \%$ bandwidth. Each curve corresponds to a different random number seed used to generate the input laser noise. 
yields a strong sensitivity to phase errors in the input laser seed; however, conventional lasers may be expected to have tighter control over phase noise than an HHG laser. In both examples, setting tolerances on noise in the seed is clearly an important aspect of designing an FEL.

\section{Conclusions}

We have presented the results of a performance study of FELs using a low-power HHG-generated EUV pulse as a seed. The FEL uses a harmonic generation FEL in the high-gain regime. For parameters which are somewhat aggressive, but reasonable, GENESIS simulations indicate robust performance and good X-ray output at $3.75 \mathrm{~nm}$. Full time-dependent simulations have been performed for several examples. The EUV-seeded FEL is compared to a two-stage harmonic cascade FEL which produces $3.75 \mathrm{~nm}$ X-rays starting from a $240 \mathrm{~nm}$ wavelength laser seed (one that could have, alternately, produced the EUV seed for the first FEL). Time-dependent simulations were used to study the sensitivity of these two types of FEL to noise in the input seed.

\section{Acknowledgements}

This work was stimulated by the mini-workshop on X-ray Drivers for FELs held at LBNL in April 2005 and attended by most of the authors. Useful discussions with fellow participants are gratefully acknowledged. This work was supported by the Director, Office of Science, High Energy Physics, US Department of Energy under Grant No. DE-FG02-04ER41289 and Contract No. DE-AC02$05 \mathrm{CH} 11231$.

\section{References}

[1] H.C. Kapteyn, M.M. Murnane, I.P. Christov, Phys. Today (March) (2005) 39.

[2] B.W. McNeil, D. Dunning, N.R. Thompson, B. Sheehy, in: Proceedings of the 28th International Free Electron Laser Conference (FEL06), Berlin, Germany, 2006, paper MOCAU03.
[3] O. Tcherbakoff, M. Labat, G. Lambert, D. Garzella, et al., in: Proceedings of the 28th International Free Electron Laser Conference (FEL06), Berlin, Germany, 2006, paper MOPPH047.

[4] L. Poletto, G. Tondello, S. De Silvestri, M. Nisoli, et al., in: Proceedings of the 28th International Free Electron Laser Conference (FEL06), Berlin, Germany, 2006, paper MOPPH028.

[5] G. Lambert, M. Bougeard, W. Boutu, B. Carré, et al., in: Proceedings of the 28th International Free Electron Laser Conference (FEL06), Berlin, Germany, 2006, paper MOPPH046.

[6] C. Pellegrini, Nucl. Instr. Meth. A 475 (2001) 1.

[7] L.-H. Yu, M. Babzien, I. Ben-Zvi, L.F. DiMauro, et al., Science 289 (2000) 932.

[8] L.-H. Yu, L.F. DiMauro, A. Doyuran, W.S. Graves, et al., Phys. Rev. Lett. 91 (2003) 074801.

[9] W.M. Fawley, W.A. Barletta, J.N. Corlett, A.A. Zholents, in: Proceedings of the Particle Accelerator Conference, 2003 (PAC2003), IEEE, vol. 2, Piscataway, NJ, 2003, 923.

[10] G. Lambert, B. Carré, M.E. Couprie, D. Garzella, et al., in: Proceedings of the 26th International Free Electron Laser Conference (FEL04), Trieste, Italy, 2004, paper MOPOS21.

[11] B.W.J. McNeil, G.R.M. Robb, N.R. Thompson, J. Jones, et al., in: Proceedings of the 27th International Free Electron Laser Conference (FEL05), Stanford, CA, USA, 2005, paper THPP025.

[12] J. Wu, P.R. Bolton, in: Proceedings of the 28th International Free Electron Laser Conference (FEL06), Berlin, Germany, 2006, paper MOPPH063.

[13] E. Takahashi, Y. Nabekawa, T. Otsuka, M. Obara, K. Midorikawa, Phys. Rev. A 66 (2002) 021802.

[14] R. Bonifacio, R. Corsini, P. Pierini, Phys. Rev. A 45 (1992) 4091.

[15] S. Reiche, Nucl. Instr. Meth. A 429 (1999) 243.

[16] G. Dattoli, A. Renieri, A. Torre, Lectures on the Free Electron Laser Theory and Related Topics, World Scientific, Singapore, 1993.

[17] R. Bonifacio, C. Pellegrini, L.M. Narducci, Opt. Commun. 50 (1984) 373.

[18] L. Giannessi, M. Quattromini, P. Musumeci, G. Sansone, S. Stagira, M. Nisoli, S. De Silvestri, in: Proceedings of the 28th International Free Electron Laser Conference (FEL06), Berlin, Germany, 2006, paper MOCAU05.

[19] J.W.G. Tisch, R.A. Smith, J.E. Muffett, M. Ciarrocca, J.P. Marangos, M.H.R. Hutchinson, Phys. Rev. A 49 (1994) R28.

[20] E.L. Saldin, E.A. Schneidmiller, M.V. Yurkov, Phys. Rev. ST Accel. Beams 9 (2006) 050702.

[21] I. Ben-Zvi, K.M. Yang, L.-H. Yu, Nucl. Inst. Meth. A 318 (1992) 726

[22] E.L. Saldin, E.A. Schneidmiller, M.V. Yurkov, Opt. Commun. 202 (2002) 169

[23] Z. Huang, in: Proceedings of the 28th International Free Electron Laser Conference (FEL06), Berlin, Germany, 2006, paper MOPPH042. 